\title{
NOTE ON A THEOREM OF FUGLEDE AND PUTNAM
}

\section{S. K. BERBERIAN}

1. An involution in a ring $A$ is a mapping $a \rightarrow a^{*}(a \in A)$ such that $a^{* *}=a,(a+b)^{*}=a^{*}+b^{*},(a b)^{*}=b^{*} a^{*}$. An element $a \in A$ is (1) normal if $a^{*} a=a a^{*}$, (2) self-adjoint if $a^{*}=a$, (3) unitary if $a^{*} a=a a^{*}=1$ ( 1 = unity element of $A$ ). We say that "Fuglede's theorem holds in $A$ " in case the relations $a \in A, a$ normal, $b \in A, b a=a b$, imply $b a^{*}$ $=a^{*} b$; briefly, $A$ is an $F T$-ring.

It follows from a theorem of $\mathrm{B}$. Fuglede that the ring $A$ of all bounded operators in a Hilbert space (hence any adjoint-containing subring thereof) is an FT-ring [3, Theorem I]. For this ring, C. R. Putnam obtained the following generalization [9, Lemma]: if $a_{1}, a_{2}$ are normal, and $b a_{1}=a_{2} b$, then $b a_{1}^{*}=a_{2}^{*} b$. A ring with involution, in which the latter theorem holds, will be called a PT-ring.

We denote by $A_{n}$ the ring of all $n \times n$ matrices $x=\left(a_{i j}\right), a_{i j} \in A$, provided with the "conjugate-transpose" involution $x^{*}=\left(a_{j k}^{*}\right)$.

Theorem 1. If $A_{2}$ is an FT-ring, then $A$ is a PT-ring.

Proof. Suppose $a_{1}, a_{2}$ are normal elements of $A$, and $b a_{1}=a_{2} b$. Define

$$
x=\left(\begin{array}{ll}
a_{1} & 0 \\
0 & a_{2}
\end{array}\right), \quad y=\left(\begin{array}{ll}
0 & 0 \\
b & 0
\end{array}\right) .
$$

Clearly $x$ is normal. Moreover,

$$
y x=\left(\begin{array}{ll}
0 & 0 \\
b a_{1} & 0
\end{array}\right), \quad x y=\left(\begin{array}{ll}
0 & 0 \\
a_{2} b & 0
\end{array}\right)
$$

thus $y x=x y$. Since Fuglede's theorem holds in $A_{2}, y x^{*}=x^{*} y$, in other words $b a_{1}^{*}=a_{2}^{*} b$.

Example 1. Let $A$ be an involutive (i.e. adjoint-containing) ring of bounded operators acting on a Hilbert space $H$. Then $A_{2}$ is an involutive ring acting on the direct sum of two copies of $H$. By Fuglede's theorem, $A_{2}$ is an FT-ring; thus $A$ is a PT-ring by Theorem 1. This is Putnam's generalization of the Fuglede theorem [9, Lemma]. The argument extends easily to cover the case that $a_{1}, a_{2}$ are possibly unbounded. The result then reads: if $b a_{1} \subset a_{2} b$ then $b a_{1}^{*} \subset a_{2}^{*} b$.

In the reverse direction, if $A$ is a PT-ring, then Fuglede's theorem

Presented to the Society, August 27, 1958; received by the editors July 28, 1958. 
holds for the diagonal normal elements of $A_{2}$; we omit the obvious proof :

Theorem 2. If $A$ is a PT-ring, and $a_{1}, a_{2} \in A$ are normal, then the commutant of the normal matrix

$$
x=\left(\begin{array}{cc}
a_{1} & 0 \\
0 & a_{2}
\end{array}\right)
$$

in $A_{2}$ is involutive; that is, the relations $y \in A_{2}, y x=x y$, imply $y x^{*}=x^{*} y$.

A ring $A$ with involution is said to satisfy the square root axiom [6, Chapter VII] in case: given any $a \in A$, there exists a self-adjoint element $r$ such that $r^{2}=a^{*} a$, and such that $r$ is in the double commutant of $a^{*} a$ (that is, the relation $b\left(a^{*} a\right)=\left(a^{*} a\right) b$ implies $b r=r b$ ). EXAmples: any $C^{*}$-algebra (see [7, Theorem 26A]); the regular ring of a finite $\mathrm{AW}^{*}$-algebra [1, Corollary 6.2]. Suppose $A$ is a ring satisfying the SR-axiom, and $a \in A$ is invertible. Write $u=a r^{-1}$, where $r$ is the self-adjoint described above; clearly $u^{*} u=u u^{*}=1$. The factorization $a=u r$ is called a "polar decomposition" for $a$.

Theorem 3. Let $A$ be a PT-ring satisfying the square-root axiom. If $a_{1}, a_{2}$ are similar normal elements, they are unitarily equivalent.

Proof. Suppose $b a_{1} b^{-1}=a_{2}$. Then $b a_{1}=a_{2} b$; since $A$ is a PT-ring, $b a_{1}^{*}=a_{2}^{*} b$, thus $a_{1} b^{*}=b^{*} a_{2}$. Let $b=u r$ be a polar decomposition. Then $a_{1}$ commutes with $b^{*} b$; for, $a_{1}\left(b^{*} b\right)=\left(a_{1} b^{*}\right) b=\left(b^{*} a_{2}\right) b=b^{*}\left(a_{2} b\right)$ $=b^{*}\left(b a_{1}\right)=\left(b^{*} b\right) a_{1}$. Hence $a_{1} r=r a_{1}$, and $a_{2}=b a_{1} b^{-1}=(u r) a_{1}\left(r^{-1} u^{*}\right)$ $=u a_{1} r r^{-1} u^{*}=u a_{1} u^{*}$.

Example 2 (Putnam). If $A$ is the ring of all bounded operators in a Hilbert space, and $a_{1}, a_{2} \in A$ are similar normal operators, then $a_{1}, a_{2}$ are unitarily equivalent by Example 1 and Theorem 3 (see [9, Theorem I]). The argument works just as well for $A$ any $C^{*}$ algebra, the point being that the elements implementing the similarity and unitary equivalence are to be drawn from $A$.

A ring $A$ with involution is said to possess a trace if there exists a mapping $a \rightarrow \operatorname{tr}(a)$ of $A$ into some abelian group, such that (1) $\operatorname{tr}(a+b)=\operatorname{tr}(a)+\operatorname{tr}(b), \quad(2) \operatorname{tr}(a b)=\operatorname{tr}(b a)$, and (3) $\quad \sum_{1}^{k} \operatorname{tr}\left(a_{i}^{*} a_{i}\right)=0$ implies $a_{1}=\cdots=a_{k}=0$.

THEOREM 4. If $A$ is a ring with involution and trace, then $A$ is a PT-ring.

Proof. Since $A_{n}$ also has a trace, defined for a matrix $x=\left(a_{i j}\right)$ by the formula $\operatorname{tr}(x)=\sum_{1}^{n} \operatorname{tr}\left(a_{i i}\right)$, it will suffice by Theorem 1 to show that $A$ is an FT-ring. Suppose $x$ is normal, and $y x=x y$. It must be 
shown that $z=y x^{*}-x^{*} y$ is 0 . We learned the ensuing argument for this from I. Kaplansky. One has

$$
\begin{aligned}
z z^{*} & =y x^{*} x y^{*}-y x^{*} y^{*} x-x^{*} y x y^{*}+x^{*} y y^{*} x \\
& =y x x^{*} y^{*}-y x^{*} y^{*} x-x^{*} x y y^{*}+x^{*} y y^{*} x \\
& =x y x^{*} y^{*}-y x^{*} y^{*} x-x x^{*} y y^{*}+x^{*} y y^{*} x .
\end{aligned}
$$

Since $\operatorname{tr}\left(x y x^{*} y^{*}\right)=\operatorname{tr}\left(y x^{*} y^{*} x\right)$, and $\operatorname{tr}\left(x x^{*} y y^{*}\right)=\operatorname{tr}\left(x^{*} y y^{*} x\right)$, one has $\operatorname{tr}\left(z z^{*}\right)=0$, hence $z=0$.

EXAmple 3. Let $A$ be a commutative ring with involution, such that $\sum_{1}^{\boldsymbol{k}} a_{i}^{*} a_{i}=0$ implies $a_{1}=\cdots=a_{k}=0$, and set $\operatorname{tr}(a)=a$. Then $A_{n}$ is a PT-ring by Theorem 4 .

EXAmPLE 4. Let $Q$ be the ring of all real quaternions $a=\alpha+\beta i+\gamma j$ $+\delta k$, with involution $a^{*}=\alpha-\beta i-\gamma j-\delta k$. One has $a^{*} a=a a^{*}=\alpha^{2}+\beta^{2}$ $+\gamma^{2}+\delta^{2}$, so that incidentally every element of $Q$ is normal. Set $\operatorname{tr}(a)=\alpha$. It results from Theorem 4 that $Q_{n}$ is a PT-ring. This is Putnam's theorem for finite-dimensional quaternionic Hilbert space, and raises the analogous question for infinite dimension.

EXAMPLE 5. Let $A$ be a homogeneous $\mathrm{AW}^{*}$-algebra of finite order $n$, so that $A=Z_{n}$, where $Z$ is the center of $A$. Let $C$ be the regular ring of $A, W$ the regular ring of $Z$; we may identify $W$ with the center of $C[1$, Theorem 9.2]. Now, $W$ has the properties in Example 3 [1, Lemma 3.4]; since $C=W_{n}$ [2, concluding remark (2)], it follows that $C$ has a $W$-valued trace. Thus $C$ is a PT-ring. See Theorem 5 for the generalization to $A$ of finite Type I.

Lemma. Suppose $A$ is the $C^{*}$-sum of a family $\left(A_{i}\right)$ of finite $A W^{*}$ algebras, $C$ is the regular ring of $A$, and $C_{i}$ is the regular ring of $A_{i}$. Then $C$ is the complete direct sum of the $C_{i}$.

Proof. According to $[5, \S 2], A$ is the set of all families $a=\left(a_{i}\right)$ with $a_{i} \in A$ and $\left\|a_{i}\right\|$ bounded; the operations in $A$ are coordinatewise. One knows from [5] that $A$ is an $\mathrm{AW}^{*}$-algebra, and is clearly of finite class, so that we may speak of its regular ring $C$.

Let $D$ be the complete direct sum of the $C_{i}$. That is, $D$ is the set of all families $x=\left(x_{i}\right)$ with $x_{i} \in C_{i}$, with the coordinatewise operations. By an easy coordinatewise argument, one sees that $D$ is regular. It must be shown that $D=C$.

We may identify $A$ as an involutive subalgebra of $D$. We shall prove $D=C$ by verifying the criterion of $[1, \S 11]$. Suppose $x, y, z \in D$, and $x^{*} x+y^{*} y+z^{*} z=1$. Then $x_{i}^{*} x_{i}+y_{i}^{*} y_{i}+z_{i}^{*} z_{i}=1$ for all $i$, hence $x_{i}, y_{i}, z_{i}$ $\in A_{i}$; since these elements all have norm $\leqq 1$, one has $x, y, z \in A$. 
Theorem 5. If $A$ is a finite $A W^{*}$-algebra of Type $I$, its regular ring $C$ possesses a center-valued trace. In particular, $C$ is a PT-ring.

Proof. Write $A$ as the $C^{*}$-sum of a family $\left(A_{i}\right)$ of homogeneous algebras, and let $C_{i}$ be the regular ring of $A_{i}$. By the Lemma, $C$ is the complete direct sum of the $C_{i}$. It follows at once that the center $W$ of $C$ is the complete direct sum of the centers $W_{i}$ of $C_{i}$. According to Example 5, $C_{i}$ has a $W_{i}$-valued trace. Then $\left(x_{i}\right) \rightarrow\left(\operatorname{tr} x_{i}\right)$ defines a $W$-valued trace on $C$, thus $C$ is a PT-ring by Theorem 4 .

It is reasonable to suppose that $C$ is a PT-ring, for any finite AW*-algebra $A$; in any case, since $A_{2}$ is $\mathrm{AW}^{*}$ with regular ring $C_{2}$ by [2], it would suffice by Theorem 1 to show that $C$ is an FT-ring.

Coroliary. $A, C$ as in Theorem 5. If $z_{1}, z_{2}$ are similar normal elements of $C$, they are unitarily equivalent.

Proof. $C$ is a PT-ring, with square root axiom [1, Corollary 6.2]; quote Theorem 3.

It results from the corollary that if two normal elements are similar via an unbounded element, they are already similar via a bounded (even unitary) element; in particular, a normal bounded element cannot be similar to a normal unbounded element. Normality is essential here, as is shown by the following example due to Jacob Feldman:

EXAmple 6. Let $A$ be the $C^{*}$-sum of denumerably many copies of the algebra $K_{2}$ of $2 \times 2$ complex matrices. $A$ may be represented as the algebra of all functions $n \rightarrow f(n)(n=1,2,3, \cdots)$, with $f(n) \in K_{2}$, $\|f(n)\|$ bounded, and operations pointwise. Since $K_{2}$ is its own regular ring, the regular ring $C$ of $A$ is the algebra of all functions $n \rightarrow f(n)$ with $f(n) \in K_{2}$. Consider the functions $f, g, h \in C$ defined by

$$
f(n)=\left(\begin{array}{ll}
0 & 0 \\
1 & 0
\end{array}\right), \quad g(n)=\left(\begin{array}{ll}
0 & 0 \\
n & 0
\end{array}\right), \quad h(n)=\left(\begin{array}{ll}
1 & 0 \\
0 & n
\end{array}\right) .
$$

Since $h(n) f(n) h(n)^{-1}=g(n)$ for all $n$, one has $h f h^{-1}=g$. Thus $f$ and $g$ are similar in $C$, even though $f$ is bounded (i.e., is an element of $A$ ) and $g$ is not bounded.

2. More on the regular ring. Throughout, $C$ denotes the regular ring of a finite $\mathrm{AW}^{*}$-algebra $A$ (of unrestricted type).

If $x \in C$, and $R P(x)=1$, then $x$ is invertible. For, $C x=C$ [1, Corollary 7.1], so there exists $y \in C$ with $y x=1$; moreover $L P(x) \sim R P(x)$ $=1$, hence $L P(x)=1$ by finiteness, $x C=C, x z=1$ for suitable $z$. Note that an $x \in C$ is invertible if and only if it is left (right) invertible.

If $x \in C$ is invertible, then $x^{*}$ is invertible, and $\left(x^{*}\right)^{-1}=\left(x^{-1}\right)^{*}$; if moreover $x$ is self-adjoint, so is $x^{-1}$. 
Lemma 1. If $x \in C, x \geqq 0$, and $x$ is invertible, then $x^{-1} \geqq 0$.

Proof. Say $x y=y x=1$, and $x=z^{*} z$ [1, Definition 6.1]. Then $\left(y z^{*}\right) z=1$ shows that $z$ is invertible (see above remarks), hence $x^{-1}$ $=\left(z^{*} z\right)^{-1}=\left(z^{-1}\right)\left(z^{-1}\right)^{*} \geqq 0$.

Lemma 2. Let $a \in A, 0 \leqq a \leqq 1$, and suppose $a$ has an inverse in $C$. Then $a^{-1} \geqq 1$.

Proof. Say $a x=x a=1$; we know $x \geqq 0$ from Lemma 1 . Write $x=y^{2}, y$ self-adjoint [1, Corollary 6.2]. Since $(a y) y=y(y a)=1, y$ is invertible, and $a y=y a=y^{-1}$. Then $a \leqq 1, y^{*} a y \leqq y^{*} y, y a y \leqq y^{2}$, $a y^{2} \leqq y^{2}, a x \leqq x, 1 \leqq x$.

Theorem 6. Suppose $x, y \in C, 0 \leqq x \leqq y$, and $x$ is invertible. Then $y$ is invertible, and $x^{-1} \geqq y^{-1} \geqq 0$.

Proof. The relation $0 \leqq x \leqq y$ implies $R P(x) \leqq R P(y)$ (see the proof of [1, Corollary 7.6]); by assumption $R P(x)=1$, hence $R P(y)=1, y$ is invertible. Write $x^{1 / 2}=w y^{1 / 2}$ with $w \in A, w^{*} w \leqq 1$ [1, Corollary 7.6]. Then $w=\left(x^{1 / 2}\right)\left(y^{1 / 2}\right)^{-1}$ is invertible in $C$, hence so is $w^{*} w$, and $\left(w^{*} w\right)^{-1}=\left(w^{-1}\right)\left(w^{-1}\right)^{*} \geqq 1$ by Lemma 2. Since $\left(x^{1 / 2}\right)^{-1}=\left(y^{1 / 2}\right)^{-1} w^{-1}$, one has $x^{-1}=\left(x^{1 / 2}\right)^{-1}\left(x^{1 / 2}\right)^{-1 *}=\left(y^{1 / 2}\right)^{-1}\left(w^{-1}\right)\left(w^{-1}\right)^{*}\left(y^{1 / 2}\right)^{-1} \geqq\left(y^{1 / 2}\right)^{-1}$ $\cdot\left(y^{1 / 2}\right)^{-1}=y^{-1}$. For a similar result of Rellich, see [4, Hilfsatz 4].

Corollary. Suppose $A$ has the property that every increasingly directed family of self-adjoint elements, which is bounded above, has a least upper bound. Then $C$ has the same property.

Proof. For ease of notation, we write the proof for sequences. Suppose $x_{i} \in C$ are self-adjoint, $x_{1} \leqq x_{2} \leqq x_{3} \leqq \cdots, y \in C$ is selfadjoint, and $x_{i} \leqq y$ for all $i$. Adding $-x_{1}$ throughout, we can assume $0 \leqq x_{i} \leqq y$. Then $1 \leqq 1+x_{1} \leqq 1+x_{2} \leqq \cdots \leqq 1+y$, hence by Theorem 6 , $1 \geqq\left(1+x_{1}\right)^{-1} \geqq\left(1+x_{2}\right)^{-1} \geqq \cdots \geqq(1+y)^{-1} \geqq 0$. But $\left(1+x_{i}\right)^{-1}$ and $(1+y)^{-1}$ belong to $A$ [1, Lemma 5.1]. Let $a \in A$ be the greatest lower bound of the $\left(1+x_{i}\right)^{-1}$; one has $0 \leqq(1+y)^{-1} \leqq a \leqq\left(1+x_{i}\right)^{-1}$. By Theorem $6, a$ has an inverse in $C$, and $1+y \geqq a^{-1} \geqq 1+x_{i}$. Evidently $a^{-1}-1$ is a least upper bound for the $x_{i}$. (EXAMPLE: $A$ any finite $W^{*}$-algebra; see [8, Theorem 1].)

Lemma. Let $z \in C$ be normal, and suppose there exists a complex number $\lambda$ such that $z-\lambda$ has an inverse in $A$. Then the relations $a \in A$, $a z=z a$, imply $a z^{*}=z^{*} a$.

Proof. (We are assuming, so to speak, that the "resolvent set" of $z$ is nonempty.) Suppose $a \in A, a z=z a$. Then $a(z-\lambda)=(z-\lambda) a$, and $z-\lambda$ is normal. Changing notation, assume $z$ invertible, $z^{-1} \in A$, 
$a z=z a$. Then $z^{-1} a=a z^{-1}$, hence by Fuglede's theorem $a\left(z^{-1}\right)^{*}=\left(z^{-1}\right)^{*} a$, $a\left(z^{*}\right)^{-1}=\left(z^{*}\right)^{-1} a, z^{*} a=a z^{*}$.

Theorem 7. Let $z \in C$ be normal, and write $z=x+i y$ with $x$ and $y$ self-adjoint. Suppose there exists a real number $\alpha$ such that $x-\alpha$ (or $y-\alpha)$ has an inverse in $A$. Then the relations $a \in A, a z=z a$, imply $a z^{*}=z^{*} a$.

Proof. Passing to $i z$ if necessary, we may suppose that it is $x-\alpha$ which has a bounded inverse. Then $(x-\alpha)^{-2}=(x-\alpha)^{-1 *}(x-\alpha)^{-1} \leqq \beta$ for a suitable real number $\beta>0$. By Theorem $6,(x-\alpha)^{2} \geqq 1 / \beta>0$. Since $y x=x y$ by normality, and $z-\alpha=(x-\alpha)+i y$, we have $(z-\alpha)^{*}$ $\cdot(z-\alpha)=(x-\alpha)^{2}+y^{2} \geqq(x-\alpha)^{2} \geqq 1 / \beta>0$. Hence $(z-\alpha)^{*}(z-\alpha)$ is invertible, and $(z-\alpha)^{-1}(z-\alpha)^{-1 *} \leqq \beta$. Therefore $(z-\alpha)^{-1} \in A \quad[1$, Lemma 5.1]; quote the lemma.

A self-adjoint $x \in C$ is semi-bounded in case there exists a real number $\beta$ such that either $x \leqq \beta$ or $x \geqq \beta$. For instance if $x \in A$ is selfadjoint, then $x \leqq\|x\|$. If $x$ is semi-bounded, say $x \geqq \beta$, then setting $\alpha=\beta-1$, one has $x-\alpha \geqq 1$, hence $x-\alpha$ has a bounded inverse (Theorem 6 , and Lemma 5.1 of [1]). Thus:

CoRollary. Let $z \in C$ be normal, and write $z=x+i y$, with $x$ and $y$ self-adjoint. Suppose either $x$ or $y$ is semi-bounded. Then the relations $a \in A, a z=z a$, imply $a z^{*}=z^{*} a$.

If $A$ has a trace (e.g. if $A$ is Type I, or is a finite $W^{*}$-algebra), it is clear that the relations $a \in A, a^{*} a \leqq a a^{*}$, imply $a^{*} a=a a^{*}$. We do not know if every finite $\mathrm{AW}^{*}$-algebra $A$ has this property, but whenever $A$ does, so does $C$ :

Theorem 8. Suppose the relations $a \in A, a^{*} a \leqq a a^{*}$, imply $a^{*} a=a a^{*}$. Then the relations $x \in C, x^{*} x \leqq x x^{*}$, imply $x^{*} x=x x^{*}$.

Proof. Suppose $x^{*} x \leqq x x^{*}$. Write $x=u r, r \geqq 0, u$ unitary [1, Corollary 7.4]. Then $x^{*} x=r^{2}$, and $x x^{*}=u r^{2} u^{*}=u\left(x^{*} x\right) u^{*}$. Setting $s=x^{*} x$, $t=x x^{*}$, we have $0 \leqq s \leqq t$, and $s, t$ are unitarily equivalent. Set $b=(1+s)^{-1}, c=(1+t)^{-1}$; clearly $b, c$ are unitarily equivalent, in fact $u s u^{*}=t$ yields $u b u^{*}=c$. Moreover $b \geqq c$ by Theorem 6 , and $b, c \in A$ [1, Lemma 5.1]. Set $a=b^{1 / 2} u^{*}$. Then $a a^{*}=b^{1 / 2} u^{*} u b^{1 / 2}=b \geqq c=u b u^{*}$ $=a^{*} a$. By the hypothesis on $A, a a^{*}=a^{*} a$, hence $b=c$, and this leads to $s=t$.

Corollary 1. Suppose the relations $a \in A, a^{*} a \leqq a a^{*}$, imply $a^{*} a$ $=a a^{*}$. If $x \in C$, and $x$ commutes with $x^{*} x$, then $x$ is normal.

Proof. By assumption $x x^{*} x=x^{*} x x$. Right-multiplying by $x^{*}$, 
$x x^{*} x x^{*}=x^{*} x x x^{*}$. Setting $r=x^{*} x, s=x x^{*}$, we have $r \geqq 0, s \geqq 0$, and $s^{2}=r s$. In particular $r s$ is self-adjoint, so that $r s=s r$. Hence by uniqueness of positive square roots, $s=\left(s^{2}\right)^{1 / 2}=(r s)^{1 / 2}=r^{1 / 2} s^{1 / 2}$ (see $\left[1\right.$, remarks following Definition 6.3]). Then $0 \leqq\left(r^{1 / 2}-s^{1 / 2}\right)^{2}$ $=r-2 r^{1 / 2} s^{1 / 2}+s=r-2 s+s=r-s$, thus $0 \leqq s \leqq r$. That is, $x x^{*} \leqq x^{*} x$, hence $x x^{*}=x^{*} x$ by Theorem 8 .

REMARK. In an infinite algebra $B$, choose $x \in B$ with $x^{*} x=1$ but $x x^{*} \neq 1$. Then $x$ commutes with $x^{*} x$, but is not normal.

Corollary 2. Suppose the relations $a \in A, a^{*} a \leqq a a^{*}$, imply $a^{*} a$ $=a a^{*}$. Then every triangular normal matrix in $C_{n}$ is diagonal.

Proof. Suppose e.g. $n=3$, and

$$
z=\left(\begin{array}{lll}
a & b & c \\
0 & d & e \\
0 & 0 & f
\end{array}\right)
$$

is a normal element of $C_{3}$. From the $1-1$ position in the relation $z^{*} z=z z^{*}$, one has $a^{*} a=a a^{*}+b b^{*}+c c^{*}$, thus $a^{*} a-a a^{*}=b b^{*}+c c^{*} \geqq 0$ [1, Theorem 6.1], $a a^{*} \leqq a^{*} a$. By Theorem 8, $a a^{*}=a^{*} a$, hence $b=c=0[1$, Lemma 3.4]. Inspection now of the 2-2 position similarly yields $e=0$. The case for general $n$ is an obvious induction.

REMARK. If $B$ is an infinite algebra, there exists a normal (even unitary) matrix

$$
x=\left(\begin{array}{ll}
a & b \\
0 & c
\end{array}\right)
$$

in $B_{2}$ with $b \neq 0$. For, choose a partial isometry $a \in A$ with $a^{*} a=1$, $a a^{*}=e \neq 1$, and set $b=1-e, c=a^{*}$.

Addenda. (1) I am indebted to J. Dixmier for calling my attention to the references [4] and [8].

(2) Recently M. Rosenblum has given a beautiful proof of the Fuglede-Putnam theorem; for bounded operators, the proof is nonspatial (see [10]).

\section{REFERENCES}

1. S. K. Berberian, The regular ring of a finite $A W^{*}$-algebra, Ann. of Math. vol. 65 (1957) pp. 224-240.

2. $\longrightarrow, N \times N$ matrices over an $A W^{*}$-algebra, Amer. J. Math. vol. 80 (1958) pp. $37-44$.

3. B. Fuglede, $A$ commutativity theorem for normal operators, Proc. Nat. Acad. Sci. vol. 36 (1950) pp. 35-40.

4. E. Heinz, Beiträge zur Störungstheorie der Spektralzerlegung, Math. Ann. vol. 123 (1951) pp. 415-438. 
5. I. Kaplansky, Projections in Banach algebras, Ann. of Math. vol. 53 (1951) pp. 235-249.

6. - Rings of operators, University of Chicago mimeographed notes, 1955.

7. L. H. Loomis, An introduction to abstract harmonic analysis, D. van Nostrand, 1953.

8. T. Ogasawara and K. Yoshinaga, Extension of 4 -application to unbounded operators, J. Sci. Hiroshima Univ. Ser. A. vol. 19 (1955) pp. 273-299.

9. C. R. Putnam, On normal operators in Hilbert space, Amer. J. Math. vol. 73 (1951) pp. 357-362.

10. M. Rosenblum, On a theorem of Fuglede and Putnam, J. London Math. Soc. vol. 33 (1958) pp. 376-377.

State University of Iowa

\section{A CORRECTION AND IMPROVEMENT OF A THEOREM ON ORDERED GROUPS}

PAUL CONRAD

In this note the notation and terminology of [1] will be used throughout. In particular, $G$ will always denote an $o$-group with well ordered rank. Let $P$ be the multiplicative group of positive rational numbers, and let $R$ be the additive group of real numbers. In [1] the proofs of Theorems 2 and 3 are incorrect. This is a result of the careless formulation of the theorems by the author. Consider the following properties of $G$.

(1) Each component $G^{\gamma} / G_{\gamma}$ of $G$ has its group of $o$-automorphisms isomorphic to a subgroup $P_{\gamma}$ of $P$.

(2) Each component $G^{\gamma} / G_{\gamma}$ of $G$ is $o$-isomorphic to a subgroup $D_{\gamma}$ of $R$, and the only $o$-automorphisms of $D_{\gamma}$ are multiplications by some elements of $P$.

(3) For each pair $\alpha \in Q$ and $\gamma \in \Gamma$, there exists a pair $m, n$ of positive integers such that $n g \alpha \equiv m g \bmod G_{\gamma}$ for all $g$ in $G^{\gamma}$.

The statements of Theorems 2 and 3 include the hypothesis (1), but (2) and (3) are actually used in the proofs. Clearly (2) implies (1).

Lemma. (a) (2) is independent of the particular choice of $D_{\gamma}$ (b) (2) implies (3). (c) (1) does not imply (2) or (3).

Proof. (a) Let $\sigma$ be an $o$-isomorphism of the subgroup $A$ of $R$ onto the subgroup $B$ of $R$, and suppose that the only $o$-automorphisms of $A$ are multiplications by some elements of $P$. If $\beta$ is an $o$-automor-

Received by the editors August 1, 1958. 